Article

\title{
Blood Pressure Abnormalities Associated with Gut Microbiota-Derived Short Chain Fatty Acids in Children with Congenital Anomalies of the Kidney and Urinary Tract
}

\author{
Chien-Ning Hsu ${ }^{1,2} \mathbb{D}$, Pei-Chen Lu ${ }^{3}$, Chih-Yao Hou ${ }^{4}$ and You-Lin Tain ${ }^{3,5, *(\mathbb{D})}$ \\ 1 Department of Pharmacy, Kaohsiung Chang Gung Memorial Hospital and College of Medicine, \\ Chang Gung University, Kaohsiung 833, Taiwan \\ 2 School of Pharmacy, Kaohsiung Medical University, Kaohsiung 807, Taiwan \\ 3 Department of Pediatrics, Kaohsiung Chang Gung Memorial Hospital and College of Medicine, \\ Chang Gung University, Kaohsiung 833, Taiwan \\ 4 Department of Seafood Science, National Kaohsiung University of Science and Technology, \\ Kaohsiung 811, Taiwan \\ 5 Institute for Translational Research in Biomedicine, Kaohsiung Chang Gung Memorial Hospital and Chang \\ Gung University, College of Medicine, Kaohsiung 833, Taiwan \\ * Correspondence: tainyl@hotmail.com; Tel.: +886-975-056-995; Fax: +886-7733-8009
}

Received: 27 June 2019; Accepted: 19 July 2019; Published: 24 July 2019

check for updates

\begin{abstract}
Both kidney disease and hypertension can originate from early life. Congenital anomalies of the kidney and urinary tract (CAKUT) are the leading cause of chronic kidney disease (CKD) in children. Since gut microbiota and their metabolite short chain fatty acids (SCFAs) have been linked to CKD and hypertension, we examined whether gut microbial composition and SCFAs are correlated with blood pressure (BP) load and renal outcome in CKD children with CAKUT. We enrolled 78 children with CKD stage G1-G4. Up to $65 \%$ of children with CAKUT had BP abnormalities on $24 \mathrm{~h}$ ambulatory blood pressure monitoring (ABPM). CKD children with CAKUT had lower risk of developing BP abnormalities and CKD progression than those with non-CAKUT. Reduced plasma level of propionate was found in children with CAKUT, which was related to increased abundance of phylum Verrucomicrobia, genus Akkermansia, and species Bifidobacterium bifidum. CKD children with abnormal ABPM profile had higher plasma levels of propionate and butyrate. Our findings highlight that gut microbiota-derived SCFAs like propionate and butyrate are related to BP abnormalities in children with an early stage of CKD. Early assessments of these microbial markers may aid in developing potential targets for early life intervention for lifelong hypertension prevention in childhood CKD.
\end{abstract}

Keywords: ambulatory blood pressure monitoring; butyrate; congenital anomalies of the kidney and urinary tract (CAKUT); cardiovascular disease; children; chronic kidney disease; gut microbiota; hypertension; propionate; short chain fatty acid

\section{Introduction}

Congenital anomalies of the kidney and urinary tract (CAKUT) refer to a various group of structural malformations that are characterized by defects in fetal kidney development [1]. CAKUT account for almost 30 percent of all anomalies identified in the prenatal period [2]. The causes of chronic kidney disease (CKD) in childhood differs from those in adults, as the largest category among children and adolescents are CAKUT [3]. CAKUT are the leading cause of end stage renal disease 
(ESRD) in the pediatric population [4,5]. Importantly, evidence is accumulating that many patients with CAKUT may progress to ESRD during adulthood [6].

Hypertension is the most commonly complication in pediatric CKD. We, and others, have shown that even in mild to moderate CKD, approximately $50 \%$ of children have blood pressure (BP) abnormalities [7,8]. Hypertension is associated with a high risk of developing cardiovascular disease (CVD) and more rapid progression of CKD $[9,10]$. Unlike adults, overt CVD hardly presents in children. Thus, surrogate markers, such as noninvasive measurement of blood pressure (BP) load, arterial stiffness, and vascular phenotype [11], are essential to manage and stratify risk for CVD in pediatric CKD. CKD and hypertension can both originate in early life [12,13]. Prematurity and low birth weight have relatively increased risk for the development of CAKUT, CKD, as well as hypertension later in life [13-15]. In view of this, a better understanding of the factors linking CAKUT to hypertension in early stage of CKD is essential to developing potential interventions to halt the growing epidemic of CKD-related diseases.

Recent studies suggest the pathogenic association between gut microbiota and CKD [16,17]. CKD can affect the microbial composition and their metabolites like short chain fatty acids (SCFAs) [18], whereas gut dysbiosis in CKD patients may increase gut microbiota-derived uremic toxins that in turn contribute to CKD progression. Although some microbial markers have been linked to hypertension [19-21], the impact of gut microbiota on BP and its association with CAKUT in children with early CKD remains largely unclear.

Given the importance of gut microbiota and their metabolites as risk factors for BP abnormalities and CVD, we analyzed data from the pediatric CKD cohort study to investigate their impact on microbial markers for CVD in children with the focus on CAKUT.

\section{Materials and Methods}

\subsection{Study Population}

From December 2016 to April 2019, we enrolled a total of 125 children and adolescents aged 3 to 18 years with CKD attending the pediatric clinic at Kaohsiung Chang Gung Memorial Hospital, a medical center in Taiwan. This prospective cohort study was approved by the Institution Review Board and Ethics Committee of Chang Gung Medical Foundation, Taoyuan, Taiwan (Permit number: 201601181A3). Our study protocol was adherent to the principle of the 1964 Declaration of Helsinki and its later amendments. Written informed consent was obtained from all participants. CKD is defined as decreased kidney function or presence of persistent kidney damage over at least three months [22]. Renal function was determined by estimated glomerular filtration rate (eGFR) using the Schwartz formula according to body height and serum creatinine ( $\mathrm{Cr}$ ) level [23]. Kidney damage refers to structural abnormalities or functional abnormalities, whether established via renal biopsy or imaging studies, or inferred from markers such as urinary sediment abnormalities or proteinuria. Patients were excluded, if they (1) were already documented as pregnant; (2) had a history of congenital heart disease; (3) had an eGFR $<15 \mathrm{~mL} / \mathrm{min} / 1.73 \mathrm{~m}^{2}$, were on dialysis maintenance, or had ever received renal transplantation; (4) were unable to complete follow-up protocol or cooperate with assessment. All participants were categorized into eGFR category G1 (eGFR $\geq 90 \mathrm{~mL} / \mathrm{min} / 1.73 \mathrm{~m}^{2}$ ), G2 (eGFR 60-89 $\mathrm{mL} / \mathrm{min} / 1.73 \mathrm{~m}^{2}$ ), G3 (eGFR 30-59 mL/min/1.73 m²), or G4 (eGFR 15-29 mL/min/1.73 m²). All recruited patients were followed-up every 6 months up to progression to ESRD. Analysis was restricted to children with a baseline eGFR $>15 \mathrm{~mL} / \mathrm{min} / 1.73 \mathrm{~m}^{2}$, measured cardiovascular surrogate markers and fecal microbiota are described in the following section. In the current study, we enrolled a total of 78 children and adolescents with CKD stage G1 to G4 with a 1 year follow-up to calculate the change of eGFR to determine CKD progression. The causes of kidney diseases were divided into two categories: CAKUT or non-CAKUT. CAKUT structural anomalies range from renal agenesis, kidney hypo-/dysplasia, horseshoe kidney, duplex collecting system, multi-cystic kidney dysplasia, posterior urethral valves, and ureter abnormalities [24]. 


\subsection{Biochemical Analysis}

Fasting plasma specimens, spot urine, and fecal samples were aliquoted and stored at $-8{ }^{\circ} \mathrm{C}$ until analysis. Blood urea nitrogen (BUN), creatinine, uric acid, glucose, total cholesterol, low-density lipoprotein (LDL), triglyceride, sodium, potassium, calcium, phosphate, hemoglobin, hematocrit, and urine total protein-to-creatinine ratio were measured by the hospital's central laboratory as described previously [8].

\subsection{Office Blood Pressure and $24 \mathrm{~h}$ Ambulatory Blood Pressure Monitoring (ABPM)}

Office BP measurements were taken at a clinic visit after 5 min sitting at rest with at least $1 \mathrm{~min}$ between recordings. The mean value was used as the participant's office BP for analysis. We used the Oscar II monitoring device (SunTech Medical, Morrisville, NC, USA) to measure BP and pulse rate at $20 \mathrm{~min}$ intervals over $24 \mathrm{~h}$. The $24 \mathrm{~h}$ ambulatory blood pressure monitoring (ABPM) data were collected for subjects aged 6-18 years, handled by an experienced specialist nurse as described previously [25]. The participants and their parents were asked to keep a diary of sleeping and waking times, as well as activities that may influence BP measurements, including exercise and stressful situations. Only measurements with a systolic BP of 50-200 $\mathrm{mm} \mathrm{Hg}$, a diastolic BP of 30-100 mm Hg, and a heart rate of 30-200 beats per minute were accepted as valid and included in analysis. An abnormal ABPM profile was determined based on (1) awake, asleep, systolic, or diastolic BP loads exceeding the 95th percentile based on gender and height using ABPM reference data [26]; (2) awake, asleep, systolic, or diastolic BP load of $25 \%$ or greater; and (3) asleep decrease of BP load by less than $10 \%$ compared with average awake BP load. Next, the ambulatory arterial stiffness index (AASI) is an index derived from $24 \mathrm{~h}$ ABPM for the evaluation of arterial stiffness [27]. The diastolic BP was plotted against systolic BP using the individual $24 \mathrm{~h} \mathrm{ABPM}$ readings to calculate the linear regression slope. The AASI was defined as one minus the regression slope [27].

\subsection{Gas Chromatography-Flame Ionization Detector (GC-FID)}

Plasma acetate, butyrate, and propionate levels were measured using gas chromatography-mass spectrometry (GCMS-QP2010; Shimadzu, Kyoto, Japan) with flame ionization detector (FID), as previously reported [28]. Separation was performed on the SGE BP GC column $(21 \times 0.5 \mu \mathrm{m}$, $30 \mathrm{~m} \times 0.53 \mathrm{~mm}$; Shimadzu GLC Ltd., Tokyo, Japan). The working solutions of acetate, butyrate, and propionate used as internal and external standards were at the concentration of $10 \mathrm{mM}$ and kept at $-20^{\circ} \mathrm{C}$ in the freezer. Dry air, nitrogen, and hydrogen were supplied to the FID at 300, 20, and $30 \mathrm{~mL} / \mathrm{min}$, respectively. An aliquot of $2 \mu \mathrm{l} \mathrm{sample} \mathrm{was} \mathrm{injected} \mathrm{into} \mathrm{the} \mathrm{column.} \mathrm{The} \mathrm{inlet} \mathrm{and}$ FID temperature were set at $200{ }^{\circ} \mathrm{C}$ and $240{ }^{\circ} \mathrm{C}$, respectively. The total running time was $17.5 \mathrm{~min}$. Analytical standard grades used as internal standards for acetate and propionate were obtained from Sigma-Aldrich (St. Louis, MO, USA) and for butyrate was from Chem Service (West Chester, PA, USA).

\subsection{Analysis of Gut Microbiota Composition}

As described previously [29], Metagenomic DNA was extracted from frozen fecal samples after centrifugation. According to the manufacturer's protocol, all polymerase chain reaction amplicons were mixed together and sent to the Biotools Co., Ltd. (Taipei, Taiwan) for sequencing using Illumina Miseq platform (Illumina, San Diego, CA, USA). The sequences were analyzed using QIIME version 1.9.1. Sequences (Illumina, San Diego, CA, USA) with a distance-based similarity of $97 \%$ or greater were grouped into operational taxonomic units (OTUs) using the USEARCH algorithm. The phylogenetic relationships were determined based on a representative sequence alignment using Fast-Tree. Shannon's index accounting for both abundance and evenness of the taxa present was analyzed by QIIME version 1.9.1. We evaluated the $\beta$-diversity changes in gut microbiota across groups by the Partial Least Squares Discriminant Analysis (PLS-DA) and the Analysis of Similarities (ANOSIM). To determine the significantly differential taxa, we applied linear discriminant analysis 
effect size (LEfSe) to compare samples between groups. The LEfSe uses linear discriminant analysis (LDA) to estimate the effect size of each differentially abundant feature [30]. The threshold of the linear discriminant was set to three.

\subsection{Statistical Analysis}

Data were expressed as medians (25th, 75th percentile) for continuous variables while categorical variables were expressed as number (\%). The Mann-Whitney U-test or Chi-square test was used to test the differences in variables between children with CAKUT and non-CAKUT. The associations between variables were examined using Pearson's correlation coefficient. A value of $p<0.05$ was considered statistically significant. Analyses were performed using the Statistical Package for the Social Sciences (SPSS) software 14.0 (SPSS Inc., Chicago, IL, USA).

\section{Results}

There was a total of 78 children and adolescents with CKD in this study, including 51 G1 subjects $(65.4 \%), 17 \mathrm{G} 2$ subjects $(21.8 \%), 16 \mathrm{G} 3$ subjects $(20.5 \%)$, and one $\mathrm{G} 4$ subject $(1.3 \%)$. Our study population was slightly predominant male $(\mathrm{M}: \mathrm{F}=1.7: 1)$. The median age was $11.2(7.4-15.2)$ years. The median eGFR was $100.3(81.5-115.7) \mathrm{mL} / \mathrm{min} / 1.73 \mathrm{~m}^{2}$, indicating most participants were in an early stage of CKD. As shown in Table 1, CAKUT account for approximately 70 percent (57/78) of patients. The rate of CKD progression in 1 year follow-up was $15.8 \%$ (9/57) in the CAKUT group, a lesser extent than the non-CAKUT group which had a rate of $33.3 \%$ (7/21). In the CAKUT group, systolic and diastolic blood pressures, body mass index, and plasma levels of creatinine and uric acid were lower in the children compared to those in adolescents in an age-dependent manner. However, CAKUT adolescents had a lower eGFR but a higher rate of CKD progression compared to children. Unlike the CAKUT group, systolic and diastolic blood pressures and body mass index were not significantly different between children and adolescents in the non-CAKUT group. Additionally, we observed that the CAKUT group had a lower rate of hypertension, lower eGFR, lower urine total protein-to-creatinine ratio, lower plasma levels of LDL and triglyceride, but higher plasma levels of calcium and phosphate compared to those with non-CAKUT. While in adolescents, most parameters were not different between the CAKUT and non-CAKUT group.

Among them, 29 cases (37.2\%) had office BP exceeding the 95th percentile for age, gender, and height. A total of 55 patients (70.5\%) aged 6-18 years had undergone $24 \mathrm{~h} \mathrm{ABPM}$ and 69\% (38/55) of them had at least one BP load abnormality (Table 2). Among them, 12 cases (31.6\%) experienced CKD progression. Conversely, none of the cases with normal ABPM developed CKD progression $(p=0.009)$. The ABPM identified $14(25 \%), 16(29 \%)$, and 15 participants (27\%) with systolic BP or diastolic BP load $>95$ th percentile at $24 \mathrm{~h}$, awake, and asleep stages, respectively. Other ABPM abnormalities included 30 patients (55\%) with BP load $\geq 25 \%$ and 29 patients (53\%) with non-dipping nocturnal SBP. The cases with SBP or DBP load $>5$ th percentile at nighttime and BP load $\geq 25 \%$ were lesser in the CAKUT vs. the non-CAKUT group. The AASI, an index of arterial stiffness, was not different between the CAKUT and non-CAKUT group.

We next analyzed plasma SCFAs levels. As shown in Table 3, children with CAKUT had lower plasma levels of propionate compared to those with non-CAKUT. However, plasma levels of acetate and butyrate were not different between the two groups. Using data pooled from all subjects, correlations between plasma SCFAs levels and biochemical data, BP load, and AASI were analyzed. We observed that plasma acetate level was positively correlated with BUN $(r=0.289, p=0.011)$, total cholesterol $(\mathrm{r}=0.278, p=0.015)$, and LDL $(\mathrm{r}=0.285, p=0.013)$. Plasma propionate level was positively correlated with SBP $(\mathrm{r}=0.288, p=0.012)$, eGFR $(\mathrm{r}=0.295, p=0.01)$, urinary total protein-to-creatinine ratio $(\mathrm{r}=0.483, p<0.001)$, total cholesterol $(\mathrm{r}=0.529, p<0.001)$, LDL $(\mathrm{r}=0.423, p<0.001)$, and uric acid $(\mathrm{r}=0.282, p=0.014)$. 
Table 1. Clinical, anthropometric and biomedical characteristics in children and adolescents with chronic kidney disease (CKD).

\begin{tabular}{|c|c|c|c|c|}
\hline \multirow[t]{3}{*}{ Group } & \multicolumn{2}{|c|}{ CAKUT } & \multicolumn{2}{|c|}{ Non-CAKUT } \\
\hline & \multicolumn{2}{|c|}{$\mathrm{N}=57$} & \multicolumn{2}{|c|}{$\mathrm{N}=21$} \\
\hline & Children & Adolescents & Children & Adolescents \\
\hline & $N=39$ & $N=18$ & $\mathrm{~N}=6$ & $\mathrm{~N}=15$ \\
\hline Gender: M:F & $24: 15$ & $11: 7$ & $5: 1$ & $9: 6$ \\
\hline \multicolumn{5}{|l|}{ CKD staging } \\
\hline Stage G1 & 33 & 6 & 5 & 7 \\
\hline Stage G2 & 3 & 9 & 0 & 5 \\
\hline Stage G3 & 3 & 2 & 1 & 3 \\
\hline Stage G4 & 0 & 1 & 0 & 0 \\
\hline Age, years & $7.5(5.5-10.3)$ & $15.8(14.1-17.5)^{\mathrm{a}}$ & $10.2(7.9-11.4)$ & $15.7(13.9-16.9)^{\mathrm{a}}$ \\
\hline Body height, percentile & $50(15-75)$ & $50(25-77.5)$ & $25(3-58.8)$ & $25(3-50)$ \\
\hline Body weight, percentile & $25(15-75)$ & $50(12-85)$ & $25(3-85)$ & $25(15-85)$ \\
\hline Systolic blood pressure, $\mathrm{mmHg}$ & $105(101-113)$ & $121(111-130)^{a}$ & $114(105-119)$ & $124(112-136)$ \\
\hline Diastolic blood pressure, $\mathrm{mmHg}$ & $69(64-73)$ & $74(71-82)^{a}$ & $75(63-82)$ & $70(64-78)$ \\
\hline Body mass index, $\mathrm{kg} \cdot \mathrm{m}^{-2}$ & $15.6(14.7-17.7)$ & $20.8(17.8-24.8)^{\mathrm{a}}$ & $18.3(13.9-23.2)$ & $20.3(18.3-23.6)$ \\
\hline Hypertension (by office BP) & $12(30 \%)$ & $6(33 \%)$ & $5(83 \%)^{b}$ & $6(40 \%)$ \\
\hline Blood urea nitrogen, $\mathrm{mg} / \mathrm{dL}$ & $13(10-16)$ & $13(12-15)$ & $14(11-20)$ & $12(11-15)$ \\
\hline Creatinine, $\mathrm{mg} / \mathrm{dL}$ & $0.5(0.42-0.56)$ & $0.86(0.64-0.97)^{a}$ & $0.37(0.19-0.75)$ & $0.73(0.58-1)^{\mathrm{a}}$ \\
\hline $\mathrm{eGFR}, \mathrm{mL} / \mathrm{min} / 1.73 \mathrm{~m}^{2}$ & $104(96-116)$ & $82(71-101)^{a}$ & $136(103-393)^{b}$ & $88(71-113)^{a}$ \\
\hline $\mathrm{CKD}$ progression & $2(5.1 \%)$ & $7(38.9 \%)^{a}$ & $1(16.7 \%)$ & $5(33.3 \%)$ \\
\hline Urine total protein-to-creatinine ratio, $\mathrm{mg} / \mathrm{g}$ & $49(34-72)$ & $71(29-172)$ & $2364(709-12,293)^{b}$ & $273(51-1282)$ \\
\hline Hemoglobin, g/dL & $13.4(12.8-14)$ & $14.2(12.2-16.2)$ & $13.4(12.7-14.5)$ & $12.9(11.9-14.2)$ \\
\hline Hematocrit, \% & $39.4(37.9-41.3)$ & $42.2(37.1-46.1)$ & $38.8(37.2-40.3)$ & $38.1(36-41.6)$ \\
\hline Total cholesterol, mg/dL & $174(155-194)$ & $146(130-166)^{\mathrm{a}}$ & $239(158-353)$ & $185(132-207)$ \\
\hline $\mathrm{LDL}, \mathrm{mg} / \mathrm{dL}$ & $89(74-110)$ & $80(59-99)$ & $140(92-252)^{b}$ & $96(69-129)$ \\
\hline Triglyceride, mg/dL & $61(43-87)$ & $65(51-106)$ & $98(91-159)^{b}$ & $66(45-156)$ \\
\hline Uric acid, mg/dL & $4.8(3.9-5.4)$ & $5.9(5.5-6.8)^{a}$ & $7.6(4.1-8.7)$ & $6.9(5.2-9)$ \\
\hline Sodium, mEq/L & $141(139-142)$ & $142(140-143)$ & $141(137-142)$ & $140(140-142)$ \\
\hline Potassium, mEq/L & $4.4(4.3-4.7)$ & $4.4(4.2-4.6)$ & $4.2(3.6-4.9)$ & $4.4(4-4.7)$ \\
\hline Calcium, mg/dL & $9.9(9.7-10.1)$ & $9.9(9.5-10.2)$ & $8.5(7.3-9.8)^{b}$ & $9.6(9.2-9.6)$ \\
\hline Phosphate, $\mathrm{mg} / \mathrm{dL}$ & $5(4.7-5.3)$ & $4.5(4.1-4.9)^{a}$ & $4.5(4.1-4.7)^{\mathrm{b}}$ & $4.3(3.9-4.4)$ \\
\hline
\end{tabular}

Data are medians (25th, 75th percentile) or $\mathrm{n}(\%) .{ }^{\mathrm{a}} p<0.05$ children vs. adolescent by the Chi-square test or Mann-Whitney U-test. ${ }^{\mathrm{b}} p<0.05$ CAKUT vs. non-CAKUT by the Chi-square test or Mann-Whitney U-test. Children $=$ up to 12 years of age. Adolescents $=$ aged 13-18 years. $\mathrm{BP}=$ blood pressure; CAKUT $=$ Congenital anomalies of the kidney and urinary tract; eGFR = estimated glomerular filtration rate; LDL = low-density lipoprotein.

Table 2. ABPM profile and arterial stiffness assessment in children with CKD.

\begin{tabular}{lcc}
\hline \multicolumn{1}{c}{ Group } & CAKUT & Non-CAKUT \\
\hline 24 h ABPM & $\mathrm{N}=37$ & $\mathrm{~N}=18$ \\
Abnormal ABPM profile (with any of the following abnormalities) & $24(65 \%)$ & $14(78 \%)$ \\
Average 24 h BP $>95$ th percentile & $7(19 \%)$ & $7(39 \%)$ \\
Average daytime BP $>95$ th percentile & $8(22 \%)$ & $8(44 \%)$ \\
Average nighttime $>95$ th percentile & $7(19 \%)$ & $8(44 \%)^{*}$ \\
BP load $\geq 25 \%$ & $16(43 \%)$ & $14(78 \%)^{*}$ \\
Nocturnal decrease of BP $<10 \%$ & $20(54 \%)$ & $9(50 \%)$ \\
Arterial stiffness assessment & & \\
AASI & $0.35(0.22-0.46)$ & $0.38(0.25-0.49)$ \\
\hline
\end{tabular}

Data are medians (25th, 75th percentile) or $\mathrm{n}(\%) .{ }^{*} p<0.05$ by the Chi-square test or Mann-Whitney U-test. ABPM $=$ ambulatory blood pressure monitoring. AASI $=$ ambulatory arterial stiffness index.

Table 3. Plasma levels of acetate, propionate, and butyrate in children with CKD.

\begin{tabular}{ccc}
\hline Plasma Level & CAKUT & Non-CAKUT \\
\hline Acetate, $\mu \mathrm{M}$ & $56.4(48.5-68.1)$ & $57.9(46-83.4)$ \\
Propionate, $\mu \mathrm{M}$ & $1.8(1.5-2.2)$ & $2.5(1.6-3.3){ }^{*}$ \\
Butyrate, $\mu \mathrm{M}$ & $1.4(1.1-1.7)$ & $1.8(1.2-2.2)$ \\
\hline
\end{tabular}

Data are medians (25th, 75 th percentile) or $\mathrm{n}(\%) .{ }^{*} p<0.05$ by the Mann-Whitney U-test. 
We found that plasma propionate level was significantly higher in children with abnormal 24 $\mathrm{h}$, daytime, and nighttime BP, and BP load than those with normal profile (Table 4). Additionally, CKD children with abnormal $24 \mathrm{~h}$, daytime, and nighttime BP in ABPM profile had a higher plasma butyrate level compared to those with a normal ABPM profile.

Table 4. Plasma short chain fatty acid (SCFA) levels vs. BP and ABPM profile in children with CKD.

\begin{tabular}{|c|c|c|c|c|}
\hline BP & $\mathbf{n}$ & Acetate & Propionate & Butyrate \\
\hline \multicolumn{5}{|l|}{$24 \mathrm{~h} \mathrm{BP}$} \\
\hline Abnormal & 14 & $65.7(49.4-73.5)$ & $2.4(1.6-3.4)$ * & $1.9(1.2-2.6)$ * \\
\hline Normal & 40 & $56.1(46-66.6)$ & $1.9(1.5-2.2)$ & $1.5(1.1-1.6)$ \\
\hline \multicolumn{5}{|l|}{ Daytime BP } \\
\hline Abnormal & 16 & 65.7 (47.3-77.2) & $2.4(1.6-3.3)$ * & $1.8(1.2-2.4)$ * \\
\hline Normal & 38 & $56.1(45.5-65.7)$ & $1.9(1.5-2.2)$ & $1.4(1.1-1.6)$ \\
\hline \multicolumn{5}{|l|}{ Nighttime BP } \\
\hline Abnormal & 15 & $68.1(50.5-79.1)$ & $2.5(1.6-3.5)$ * & $1.9(1.2-2.5)$ * \\
\hline Normal & 39 & $55.8(45.9-65.3)$ & $1.8(1.5-2.2)$ & $1.4(1.1-1.6)$ \\
\hline \multicolumn{5}{|l|}{ BP load } \\
\hline Abnormal & 30 & $59.5(46.1-73.5)$ & $2.2(1.6-2.8)$ * & $1.4(1.1-1.9)$ \\
\hline Normal & 24 & $52.5(46.2-65.2)$ & $1.8(1.4-2.1)$ & $1.5(1.2-1.6)$ \\
\hline \multicolumn{5}{|l|}{$\begin{array}{l}\text { Night } \\
\text { dipping }\end{array}$} \\
\hline Abnormal & 29 & $58.9(48-75.3)$ & $2(1.6-2.6)$ & $1.3(0.8-1.7)$ \\
\hline Normal & 25 & $55.8(44.5-66.2)$ & $1.8(1.5-2.4)$ & $1.5(1.3-1.9)$ \\
\hline \multicolumn{5}{|l|}{ ABPM profile } \\
\hline Abnormal & 38 & $59(46.4-68.6)$ & $2(1.7-2.7)$ & $1.4(1-1.8)$ \\
\hline Normal & 16 & $54.2(42.7-64.5)$ & $1.8(1.3-2.2)$ & $1.5(1.3-1.8)$ \\
\hline \multicolumn{5}{|l|}{ Office BP } \\
\hline Abnormal & 29 & $54.3(49.4-68.6)$ & $2(1.7-2.7)$ & $1.5(1.2-1.9)$ \\
\hline Normal & 47 & $56.9(46.5-71.5)$ & $1.8(1.5-2.2)$ & $1.4(1-1.7)$ \\
\hline
\end{tabular}

Data are medians (25th, 75 th percentile) or $\mathrm{n}(\%) .{ }^{*} p<0.05$ by the Mann-Whitney U-test.

We further analyzed gut microbiota composition of children and adolescents with CKD. The Shannon index, an index of $\alpha$-diversity, was analyzed to determine species richness and it was found that there was no significant difference between the CAKUT and non-CAKUT group (Figure $1 \mathrm{~A} ; p=0.101$ ). The $\beta$-diversity analysis indicates the extent of similarity between microbial communities. The score plots of PLS-DA analysis showed that two groups were not well-separated (Figure 1B) and the ANOSIM analysis between the CAKUT and non-CAKUT group did not reach significance $(p=0.069)$. We observed that the main phyla were Firmicutes, Bacteroidetes, Actinobacteria, Proteobacteria, and Verrucomicrobia (Figure 1C). At the phylum level, the abundance of Verrucomicrobia was higher in the CAKUT vs. the non-CAKUT group $(p<0.01)$. The Firmicutes to Bacteroidetes ratio, a microbial biomarker for hypertension [19], was not different between children with CAKUT and those with non-CAKUT (Figure 1D). 
A
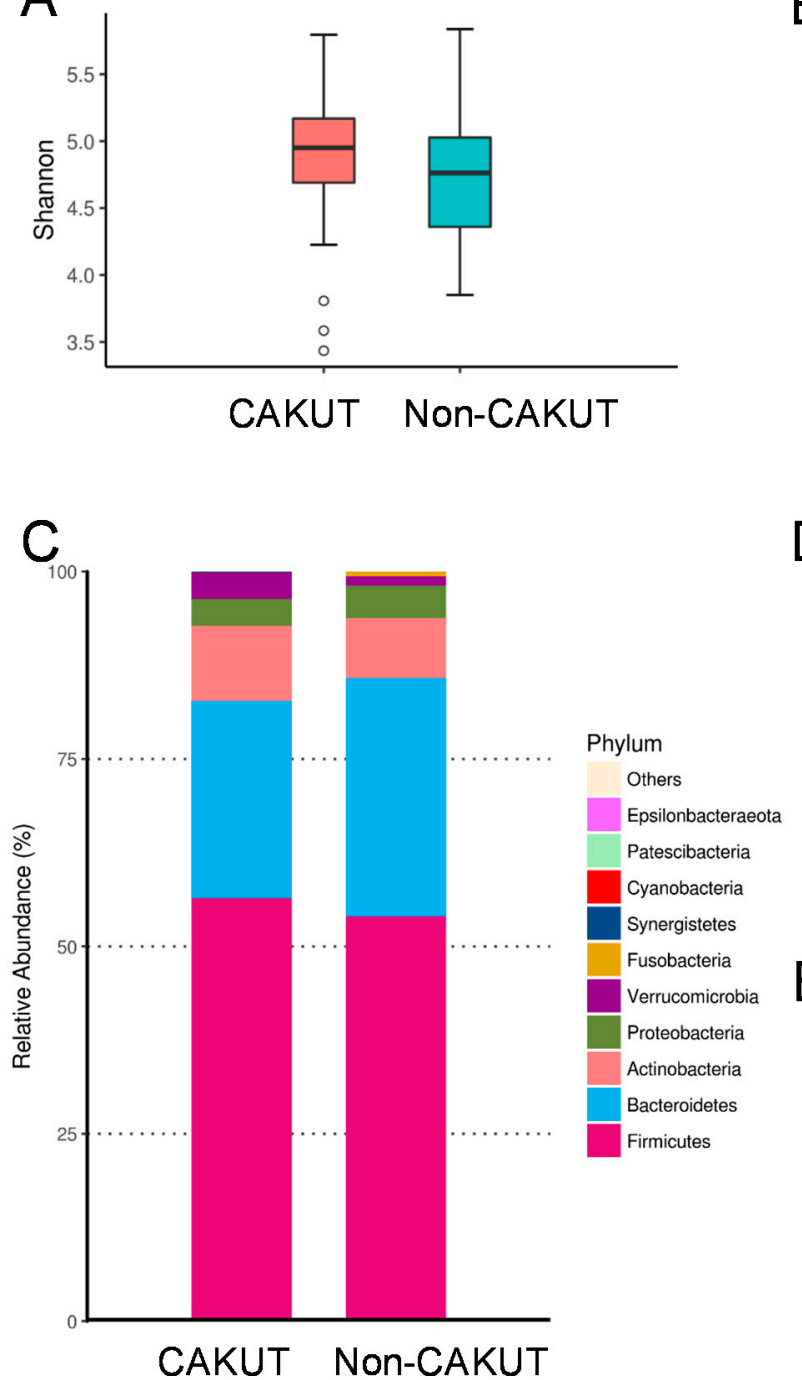
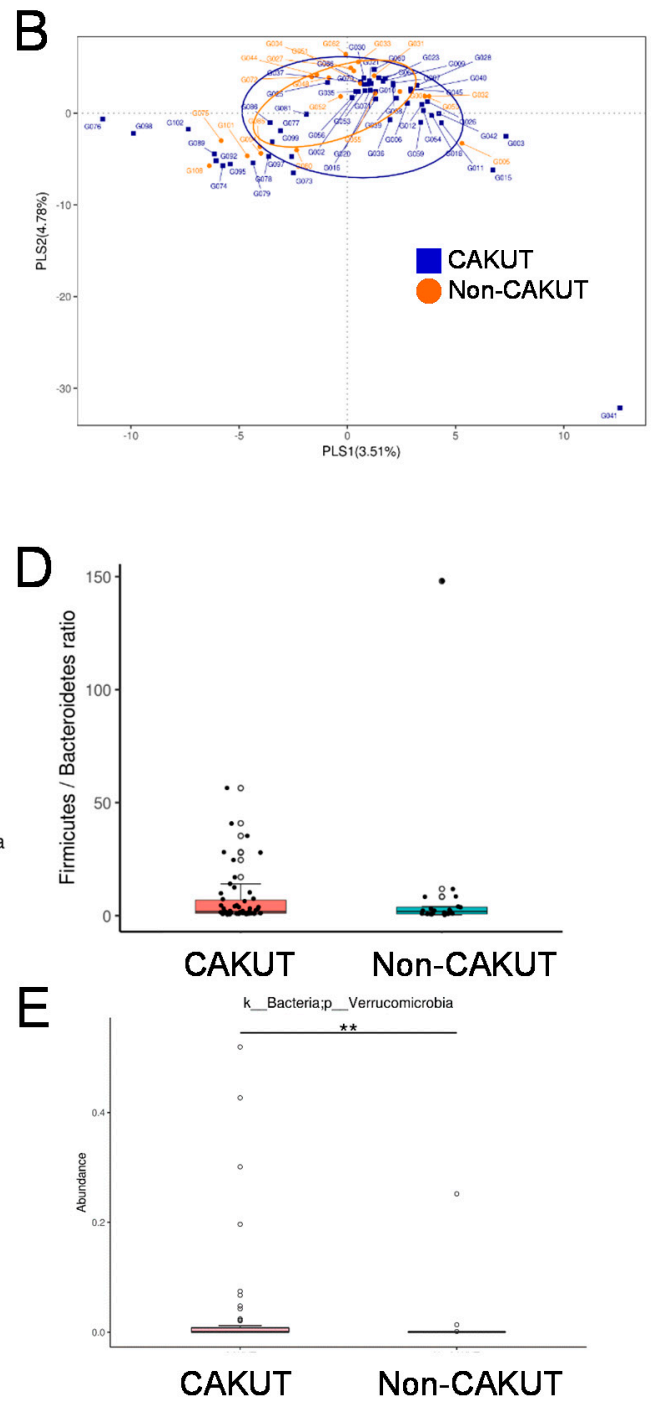

Figure 1. (A) Variation in fecal bacterial $\alpha$-diversity analyzed by the Shannon's diversity indexes. (B) $\beta$-diversity changes in gut microbiota across groups by the Partial Least Squares Discriminant Analysis (PLS-DA). (C) Relative abundance of the top 10 phylum of the gut microbiota between the CAKUT and non-CAKUT group. (D) Ratio of Firmicutes to Bacteroidetes (F/B), as a marker of gut dysbiosis. (E) The abundance of phylum Verrucomicrobia in CKD children with CAKUT vs. non-CAKUT.

The double asterisk indicates $p<0.01$.

At the genus level (Figure 2A), the abundances of the top 10 genera were not different between the CAKUT and non-CAKUT group. Additionally, we performed the LEfSe algorithm to identify metagenomic biomarkers (Figure 2B). Our results identified genera Akkermansia, Eubacterium, Ruminococcus, Clostridium, and Romboutsia in the CAKUT group are detected by LEfSe with a high LDA score (more than three orders of magnitude), reflecting marked abundance in CAKUT and low abundance in the non-CAKUT group. Children with CAKUT had decreased abundance of genus Phascolarctobacterium ( $p=0.044$ ) (Figure 2C). Additionally, the CAKUT group showed increased species Bifidobacterium bifidum $(p=0.012$ ) (Figure 2D), but decreased abundance of species Ruminococcus_sp_N15MGS_57 ( $p=0.016)$ (Figure 2E). 
A

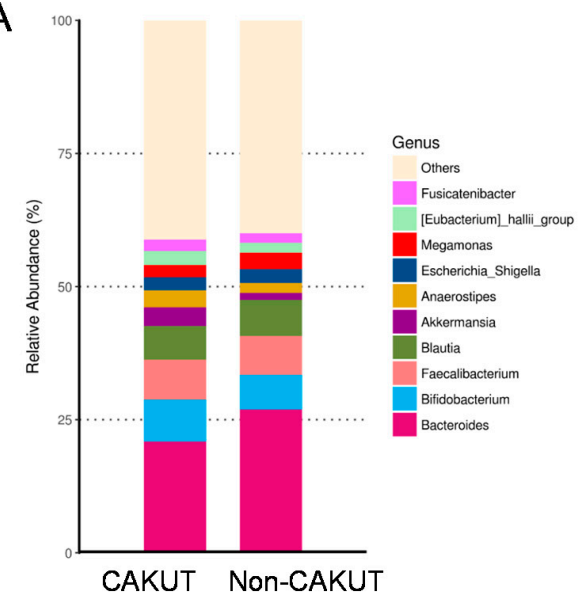

C

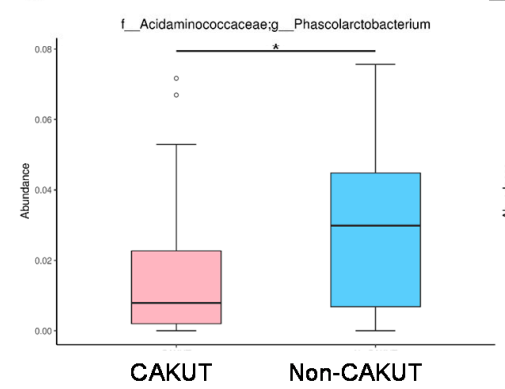

D

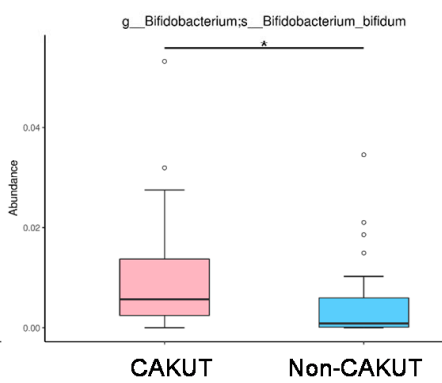

B

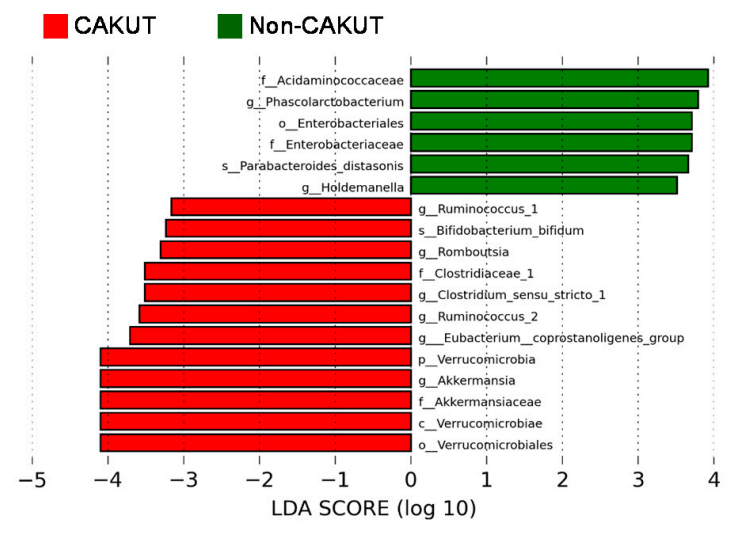

$\mathrm{E}$

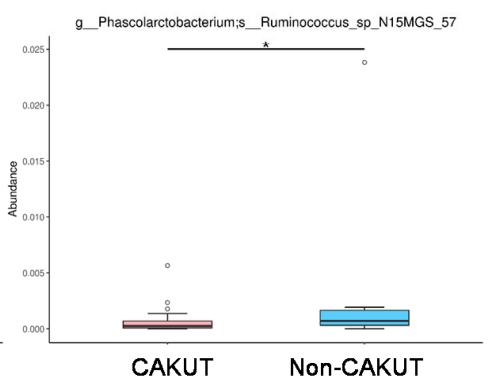

Figure 2. (A) Relative abundance of the top 10 genera of the gut microbiota between the CAKUT and non-CAKUT group. (B) Linear discriminant analysis effect size (LEfSe) to identify the taxa that were significantly different between the CAKUT and non-CAKUT group. The threshold of the linear discriminant was set to 3. (C) The abundance of genus Phascolarctobacterium, (D) species Bifidobacterium bifidum, and (E) species Ruminococcus_sp_N15MGS_57 in CKD children with CAKUT vs. non-CAKUT. The asterisk indicates $p<0.05$.

\section{Discussion}

Our study describes, for the first time, that gut microbiota-derived SCFAs link BP abnormalities to CAKUT in children and adolescents with an early stage of CKD. The key findings can be summarized as follows: (1) Up to $69 \%$ of children and adolescents with CKD stage G1-G4 had BP abnormalities in ABPM; (2) The rate of CKD progression in the 1 year follow-up was $15.8 \%$ and $33.3 \%$ in the CAKUT and non-CAKUT group, respectively; (3) Within the CAKUT group, adolescents had a lower eGFR and higher rate of CKD progression compared to children; (4) CKD children with CAKUT had lower risk of developing nighttime hypertension and BP load $\geq 25 \%$ than the non-CAKUT group; (5) Children with CAKUT had lower plasma levels of propionate compared to those with non-CAKUT; (6) CKD children with an abnormal ABPM profile had higher plasma levels of propionate and butyrate; and (7) CKD children with CAKUT had a higher abundance of phylum Verrucomicrobia, genus Akkermansia, and species Bifidobacterium bifidum than those with non-CAKUT.

In the current study, approximately 70\% of CKD children had BP abnormalities in ABPM, while those with CAKUT displayed BP abnormalities to a lesser extent. This result ties in well with previous studies wherein hypertension is highly prevalent in CKD children, even in an early stage of CKD [8-10,31]. The present study also confirmed the notion that ABPM is superior to office BP in identifying children with BP abnormalities [11]. Children with CAKUT are expected to progress to ESRD because the congenital reduction in nephron number eventually overloads the remaining nephrons [1]. In the ItalKid Study, in a population-based registry of children with CAKUT, the risk of progressing to ESRD by the age of 20 was $68 \%$ [32]. In our overall study population, the 1 year CKD progression rate 
was $20.5 \%$, which is comparable to that reported previously $[33,34]$. Of note is that children with CAKUT had a lower CKD progression rate compared to those in the non-CAKUT group. Our data supports the notion that many children with mild to moderate renal hypoplasia/dysplasia can maintain stable CKD during childhood and progress slowly to ESRD until adulthood [6]. Additionally, our data showed that children with an abnormal ABPM profile are prone to experience CKD progression. Hypertension is a risk factor for CKD progression [33], so it is not surprising to see CAKUT associated with low occurrence of BP abnormalities and CKD progression. Consistent with a previous study showing that patients with CAKUT survived longer than non-CAKUT controls due to lower cardiovascular mortality [5,6], our results showed the proportion of BP abnormalities and CKD progression was lower in children with CAKUT than in those with non-CAKUT. We previously showed that AASI, an index of arterial stiffness, was correlated with BP abnormalities in children with an early stage of CKD [25]. Nevertheless, we found no difference in AASI between CAKUT and non-CAKUT.

According to our data, children with CAKUT had increased abundance of phylum Verrucomicrobia, genera Akkermansia, Ruminococcus, Clostridium, and Romboutsia, and species Bifidobacterium bifidum. Akkermansia, a genus in the phylum Verrucomicrobia, is known as a beneficial gut microbe [35]. Similarly, Bifidobacterium bifidum has been shown to benefit cardiovascular health [36]. A previous study reported that the abundance of genera Akkermansia, Ruminococcus, Clostridium, and Roseburia were deficient in mice with hypertension [37]. Overall these observations suggest that these certain bacteria populations might have beneficial properties in early-stage CAKUT children to halt the progression of hypertension and CKD. The Firmicutes to Bacteroidetes ratio has been used as a microbial marker for hypertension $[19,20]$. However, we did not find a difference in the Firmicutes to Bacteroidetes ratio between CAKUT and non-CAKUT, regardless of non-CAKUT being associated with increased occurrence of BP abnormalities. This is possibly because we analyzed CKD children preceding hypertension onset but not in the stage of established hypertension. Another possible reason for not reaching significance may be due to a small sample size with insufficient power.

Emerging evidence support that SCFAs link gut microbiota to BP regulation [17-21]. From our results it is clear that elevated plasma propionate level is relevant to children with non-CAKUT as well as an abnormal ABPM profile. Intriguingly, SCFAs are generally known to induce vasorelaxation [37]. Accordingly, elevated propionate level is presumed to reduce rather than induce BP. However, a previous study demonstrated that propionate can modify renin release and increase BP in an olfactory receptor 78 (Olfr78)-dependent manner [38]. Additionally, elevated propionate level is related to hypertension in a salt-sensitive hypertension rat model [39]. Our data showed propionate level was positively correlated with several risk factors related to hypertension, such as urinary total protein-to-creatinine ratio, total cholesterol, LDL, and uric acid. Thus, whether propionate plays a beneficial or detrimental role in the development of hypertension in children with an early stage of CKD deserves further clarification. The succinate pathway is the dominant route for the generation of propionate, which is found mainly in Phascolarctobacterium spp. [40]. Since the abundance of genus Phascolarctobacterium was higher in the non-CAKUT vs. CAKUT group, and non-CAKUT had higher propionate levels than that in CAKUT, whether targeting of genus Phascolarctobacterium to lower propionate level may aid in protecting non-CAKUT children against hypertension awaits further elucidation.

Although plasma butyrate level was not different between the CAKUT and non-CAKUT group, its level is related to high BP load. The major genera of butyrate-producing microbes include Coprococcus, Facecalibacterium, Eubacterium, and Roseburia [41]. Our results demonstrated that these butyrate-producing microbes were not different between the CAKUT and non-CAKUT group. Like propionate, butyrate has been reported to display a vasodilatory property [37]. However, the role of butyrate in hypertension remains controversial. Although low abundance of butyrate-producing microbes and blood butyrate level were found in spontaneously hypertensive rats [19,42], butyrate like propionate is a ligand for Olfr78 that can induce renin release and elevate BP [38]. Additional studies are required to clarify whether these alterations of gut microbiota-derived SCFAs are involved in the development of hypertension and CKD progression in patients with early stage of CKD. Emerging 
evidence suggests several mechanisms by which intestinal dysbiosis associated with CKD contributes to cardiovascular disease, such as defects in intestinal barrier function, inflammation, and reduced clearance of microbiota-derived uremic toxins [16,43]. Conversely, microbiota-targeted interventions have been shown to improve cardiovascular outcomes in CKD $[18,44]$. Despite recent advances in exploring molecular mechanisms of CAKUT [1,24], the pathogenic link between gut dysbiosis and cardiovascular morbidity in CKD children with CAKUT remains largely unknown. In the present study, we see great opportunities for the potential use of gut microbiota and its derived SCFAs as markers for BP abnormalities in children with CAKUT. The possibility of microbiota-targeted interventions in preventing hypertension and cardiovascular mobility in CKD youths with CAKUT warrants further investigation.

Our study has several limitations. First, the 1 year follow-up period is short. More CV assessments with longer follow-ups are required in view of the long-term nature of childhood CKD. Second, we acknowledge that our sample of CKD children from one hospital may not be representative of the population as a whole. Larger numbers of patients recruited via multi-center cohorts may be warranted in the future to elucidate the true relationship. Third, we found a correlation between certain taxa and $\mathrm{BP}$ abnormalities but we do not reveal the pathophysiological mechanism by which those specific taxa contribute to the development of hypertension. Additionally, the composition of gut microbiota can change in an age-dependent manner. Future studies should aim to replicate results in a larger cohort comparing CAKUT with non-CAKUT that are matched by age. Last, we used ABPM reference values from studies performed in Germany [26]. Ethnic differences should be considered.

\section{Conclusions}

In conclusion, BP abnormalities are highly prevalent in CAKUT, the most important cause of CKD in children. Our results cast a new light on the link between gut microbiota, SCFAs, BP load, and CKD progression in youths with early stage of CKD. Since both kidney disease and hypertension can originate in earliest childhood, early detection of microbial markers related to BP abnormalities and CKD progression may aid in improving cardiovascular outcome in children with CAKUT.

Author Contributions: C.-N.H.: contributed to concept generation, data interpretation, drafting of the manuscript, critical revision of the manuscript and approval of the article; P.-C.L.: contributed to data interpretation, critical revision of the manuscript and approval of the article; C.-Y.H.: contributed to methodology and approval of the article; Y.-L.T.: contributed to concept generation, data interpretation, drafting of the manuscript, critical revision of the manuscript and approval of the article.

Funding: This work was supported by grants CORPG8F1031, CORPG8F1032, and CORPG8F1033 from the Kaohsiung Chang Gung Memorial Hospital, Taiwan.

Acknowledgments: We would like to thank Yu-Lun Kuo at BIOTOOLS Co., Ltd. in Taiwan for kindly supporting analysis of microbiome NGS data.

Conflicts of Interest: The authors declare no conflict of interest.

\section{References}

1. Nicolaou, N.; Renkema, K.Y.; Bongers, E.M.; Giles, R.H.; Knoers, N.V. Genetic, environmental, and epigenetic factors involved in CAKUT. Nat. Rev. Nephrol. 2015, 11, 720-731. [CrossRef] [PubMed]

2. Queisser-Luft, A.; Stolz, G.; Wiesel, A.; Schlaefer, K.; Spranger, J. Malformations in newborn: Results based on 30,940 infants and fetuses from the Mainz congenital birth defect monitoring system (1990-1998). Arch. Gynecol. Obstet. 2002, 266, 163. [CrossRef] [PubMed]

3. Harambat, J.; van Stralen, K.J.; Kim, J.J.; Tizard, E.J. Epidemiology of chronic kidney disease in children. Pediatr. Nephrol. 2012, 27, 363-373. [CrossRef] [PubMed]

4. Saran, R.; Li, Y.; Robinson, B.; Ayanian, J.; Balkrishnan, R.; Bragg-Gresham, J.; Chen, J.T.; Cope, E.; Gipson, D.; $\mathrm{He}, \mathrm{K}$;; et al. US renal data system 2014 annual data report: Epidemiology of kidney disease in the United States. Am. J. Kidney Dis. 2015, 65, A7. [CrossRef] [PubMed] 
5. Chou, H.H.; Chiou, Y.Y.; Chiou, Y.H.; Tain, Y.L.; Wang, H.H.; Yu, M.C.; Hsu, C.C.; Lin, C.Y. Mortality Risks among Various Primary Renal Diseases in Children and Adolescents on Chronic Dialysis. J. Clin. Med. 2018, 7, 414. [CrossRef]

6. Wuhl, E.; Van Stralen, K.J.; Verrina, E.; Bjerre, A.; Wanner, C.; Heaf, J.G.; Zurriaga, O.; Hoitsma, A.; Niaudet, P.; Palsson, R.; et al. Timing and outcome of renal replacement therapy in patients with congenital malformations of the kidney and urinary tract. Clin. J. Am. Soc. Nephrol. 2013, 8, 67-74. [CrossRef] [PubMed]

7. Flynn, J.T.; Mitsnefes, M.; Pierce, C.; Cole, S.R.; Parekh, R.S.; Furth, S.L.; Warady, B.A. Chronic Kidney Disease in Children Study Group: Blood pressure in children with chronic kidney disease: A report from the Chronic Kidney Disease in Children study. Hypertension 2008, 52, 631-637. [CrossRef]

8. Hsu, C.N.; Lu, P.C.; Lo, M.H.; Lin, I.C.; Chang-Chien, G.P.; Lin, S.; Tain, Y.L. Gut Microbiota-Dependent Trimethylamine N-Oxide Pathway Associated with Cardiovascular Risk in Children with Early-Stage Chronic Kidney Disease. Int. J. Mol. Sci. 2018, 19, 3699. [CrossRef]

9. Wühl, E.; Trivelli, A.; Picca, S.; Litwin, M.; Peco-Antic, A.; Zurowska, A.; Testa, S.; Jankauskiene, A.; Emre, S.; Caldas-Afonso, A.; et al. ESCAPE Trial Group: Strict blood-pressure control and progression of renal failure in children. N. Engl. J. Med. 2009, 361, 1639-1650.

10. Mitsnefes, M.M. Cardiovascular disease in children with chronic kidney disease. J. Am. Soc Nephrol. 2012, 23, 578-585. [CrossRef]

11. Urbina, E.M.; Williams, R.V.; Alpert, B.S.; Collins, R.T.; Daniels, S.R.; Hayman, L.; Jacobson, M.; Mahoney, L.; Mietus-Snyder, M.; Rocchini, A.; et al. American Heart Association Atherosclerosis, Hypertension, and Obesity in Youth Committee of the Council on Cardiovascular Disease in the Young. American Heart Association Atherosclerosis, Hypertension, and Obesity in Youth Committee of the Council on Cardiovascular Disease in the Young. Noninvasive assessment of subclinical atherosclerosis in children and adolescents: Recommendations for standard assessment for clinical research: A scientific statement from the American Heart Association. Hypertension 2009, 54, 919-950. [PubMed]

12. Ingelfinger, J.R.; Kalantar-Zadeh, K.; Schaefer, F.; World Kidney Day Steering Committee. World Kidney Day 2016: Averting the legacy of kidney disease-focus on childhood. Pediatr. Nephrol. 2016, 31, 343-348. [CrossRef] [PubMed]

13. Tain, Y.L.; Hsu, C.N. Developmental Origins of Chronic Kidney Disease: Should We Focus on Early Life? Int. J. Mol. Sci. 2017, 18, 381. [CrossRef] [PubMed]

14. Luyckx, V.A.; Bertram, J.F.; Brenner, B.M.; Fall, C.; Hoy, W.E.; Ozanne, S.E.; Vikse, B.E. Effect of fetal and child health on kidney development and long-term risk of hypertension and kidney disease. Lancet 2013, 382, 273-283. [CrossRef]

15. Tain, Y.L.; Luh, H.; Lin, C.Y.; Hsu, C.N. Incidence and Risks of Congenital Anomalies of Kidney and Urinary Tract in Newborns: A Population-Based Case-Control Study in Taiwan. Medicine 2016, 95, e2659. [CrossRef] [PubMed]

16. Jovanovich, A.; Isakova, T.; Stubbs, J. Microbiome and Cardiovascular Disease in CKD. Clin. J. Am. Soc. Nephrol. 2018, 13, 1598-1604. [CrossRef] [PubMed]

17. Meijers, B.; Jouret, F.; Evenepoel, P. Linking gut microbiota to cardiovascular disease and hypertension: Lessons from chronic kidney disease. Pharmacol. Res. 2018, 133, 101-107. [CrossRef]

18. Felizardo, R.J.F.; Watanabe, I.K.M.; Dardi, P.; Rossoni, L.V.; Câmara, N.O.S. The interplay among gut microbiota, hypertension and kidney diseases: The role of short-chain fatty acids. Pharmacol. Res. 2019, 141, 366-377. [CrossRef]

19. Yang, T.; Santisteban, M.M.; Rodriguez, V.; Li, E.; Ahmari, N.; Carvajal, J.M.; Zadeh, M.; Gong, M.; Qi, Y.; Zubcevic, J.; et al. Gut dysbiosis is linked to hypertension. Hypertension 2015, 65, 1331-1340. [CrossRef]

20. Al Khodor, S.; Reichert, B.; Shatat, I.F. The microbiome and blood pressure: Can microbes regulate our blood pressure? Front. Pediatr. 2017, 5, 138. [CrossRef]

21. Chambers, E.S.; Preston, T.; Frost, G.; Morrison, D.J. Role of Gut Microbiota-Generated Short-Chain Fatty Acids in Metabolic and Cardiovascular Health. Curr. Nutr. Rep. 2018, 7, 198-206. [CrossRef] [PubMed]

22. Levin, A.; Stevens, P.E.; Bilous, R.W.; Coresh, J.; De Francisco, A.L.M.; De Jong, P.E.; Griffith, K.E.; Hemmelgarn, B.R.; Iseki, K.; Lamb, E.J. Kidney Disease: Improving Global Outcomes (KDIGO) CKD Work Group. KDIGO 2012 clinical practice guideline for the evaluation and management of chronic kidney disease. Kidney Int. Suppl. 2013, 3, 1-150. 
23. Schwartz, G.J.; Muñoz, A.; Schneider, M.F.; Mak, R.H.; Kaskel, F.; Warady, B.A.; Furth, S.L. New equations to estimate GFR in children with CKD. J. Am. Soc. Nephrol. 2009, 20, 629-637. [CrossRef] [PubMed]

24. Renkema, K.Y.; Winyard, P.J.; Skovorodkin, I.N.; Levtchenko, E.; Hindryckx, A.; Jeanpierre, C.; Weber, S.; Salomon, R.; Antignac, C.; Vainio, S.; et al. Novel perspectives for investigating congenital anomalies of the kidney and urinary tract (CAKUT). Nephrol. Dial. Transplant. 2011, 26, 3843-3851. [CrossRef] [PubMed]

25. Lin, I.C.; Hsu, C.N.; Lo, M.H.; Chien, S.J.; Tain, Y.L. Low urinary citrulline/arginine ratio associated with blood pressure abnormalities and arterial stiffness in childhood chronic kidney disease. J. Am. Soc. Hypertens. 2016, 10, 115-123. [CrossRef] [PubMed]

26. Wuhl, E.; Witte, K.; Soergelm, M.; Mehls, O.; Schaefer, F.; German Working Group on Pediatric Hypertension. Distribution of 24-h ambulatory blood pressure in children: Normalized reference values and role of body dimensions. J. Hypertens. 2002, 20, 1995-2007. [CrossRef] [PubMed]

27. Kollias, A.; Stergiou, G.S.; Dolan, E.; O’Brien, E. Ambulatory arterial stiffness index: A systematic review and meta-analysis. Atherosclerosis 2012, 224, 291-301. [CrossRef] [PubMed]

28. Hsu, C.N.; Lin, Y.L.; Hou, C.Y.; Tain, Y.L. Maternal Administration of Probiotic or Prebiotic Prevents Male Adult Rat Offspring against Developmental Programming of Hypertension Induced by High Fructose Consumption in Pregnancy and Lactation. Nutrients 2018, 10, 1229. [CrossRef]

29. Hsu, C.N.; Chang-Chien, G.P.; Lin, S.; Lo, M.H.; Tain, Y.L. Targeting on gut microbial metabolite trimethylamine-N-oxide (TMAO) and short chain fatty acid to prevent maternal high-fructose diet-induced developmental programming of hypertension in adult male offspring. Mol. Nutr. Food Res. 2019, in press. [CrossRef]

30. Fisher, R.A. The use of multiple measurements in taxonomic problems. Ann. Eugenics. 1936, 7, 179-188. [CrossRef]

31. Chou, H.H.; Lin, C.Y.; Chiou, Y.H.; Tain, Y.L.; Wang, Y.F.; Wang, H.H.; Chiou, Y.Y. Clinical characteristics and prevalence of complications of chronic kidney disease in children: The Taiwan Pediatric Renal Collaborative study. Pediatr. Nephrol. 2016, 31, 1113-1120. [CrossRef] [PubMed]

32. Ardissino, G.; Daccò, V.; Testa, S.; Bonaudo, R.; Claris-Appiani, A.; Taioli, E.; Marra, G.; Edefonti, A.; Fabio Sereni on Behalf of the ItalKid Project. Epidemiology of chronic renal failure in children: Data from the ItalKid project. Pediatrics 2003, 111, e382-e387. [CrossRef] [PubMed]

33. Staples, A.O.; Greenbaum, L.A.; Smith, J.M.; Gipson, D.S.; Filler, G.; Warady, B.A.; Martz, K.; Wong, C.S. Association between clinical risk factors and progression of chronic kidney disease in children. Clin. J. Am. Soc. Nephrol. 2010, 5, 2172-2179. [CrossRef] [PubMed]

34. Chiou, Y.Y.; Lin, C.Y.; Chen, M.J.; Chiou, Y.H.; Wang, Y.F.; Wang, H.H.; Tain, Y.L.; Chou, H.H. Etiology and pediatric chronic kidney disease progression: Taiwan Pediatric Renal Collaborative Study. J. Formos. Med. Assoc. 2016, 115, 752-763. [CrossRef] [PubMed]

35. Cani, P.D.; de Vos, W.M. Next-Generation Beneficial Microbes: The Case of Akkermansia muciniphila. Front. Microbiol. 2017, 8, 1765. [CrossRef] [PubMed]

36. Li, J.; Zhao, F.; Wang, Y.; Chen, J.; Tao, J.; Tian, G.; Wu, S.; Liu, W.; Cui, Q.; Geng, B. Gut microbiota dysbiosis contributes to the development of hypertension. Microbiome 2017, 5, 14. [CrossRef] [PubMed]

37. Pluznick, J.L. Microbial short chain fatty acids and blood pressure regulation. Curr. Hypertens. Rep. 2017, 19, 25. [CrossRef]

38. Pluznick, J.L.; Protzko, R.J.; Gevorgyan, H.; Peterlin, Z.; Sipos, A.; Han, J.; Brunet, I.; Wan, L.X.; Rey, F.; Wang, T.; et al. Olfactory receptor responding to gut microbiota-derived signals plays a role in renin secretion and blood pressure regulation. Proc. Natl. Acad. Sci. USA 2013, 110, 4410-4415. [CrossRef]

39. Bier, A.; Braun, T.; Khasbab, R.; Di Segni, A.; Grossman, E.; Haberman, Y.; Leibowitz, A. A High Salt Diet Modulates the Gut Microbiota and Short Chain Fatty Acids Production in a Salt-Sensitive Hypertension Rat Model. Nutrients 2018, 10, 1154. [CrossRef]

40. Reichardt, N.; Duncan, S.H.; Young, P.; Belenguer, A.; McWilliam Leitch, C.; Scott, K.P.; Flint, H.J.; Louis, P. Phylogenetic distribution of three pathways for propionate production within the human gut microbiota. ISME J. 2014, 8, 1323-1335. [CrossRef]

41. Ríos-Covián, D.; Ruas-Madiedo, P.; Margolles, A.; Gueimonde, M.; de Los Reyes-Gavilán, C.G.; Salazar, N. Intestinal Short Chain Fatty Acids and their Link with Diet and Human Health. Front. Microbiol. 2016, 7, 185. [CrossRef] [PubMed] 
42. Yang, T.; Magee, K.L.; Colon-Perez, L.M.; Larkin, R.; Liao, Y.S.; Balazic, E.; Cowart, J.R.; Arocha, R.; Redler, T.; Febo, M.; et al. Impaired butyrate absorption in the proximal colon, low serum butyrate and diminished central effects of butyrate on blood pressure in spontaneously hypertensive rats. Acta Physiol. 2019, 226, e13256. [CrossRef] [PubMed]

43. Meijers, B.; Evenepoel, P.; Anders, H.J. Intestinal microbiome and fitness in kidney disease. Nat. Rev. Nephrol. 2019. [CrossRef] [PubMed]

44. Chen, Y.Y.; Chen, D.Q.; Chen, L.; Liu, J.R.; Vaziri, N.D.; Guo, Y.; Zhao, Y.Y. Microbiome-metabolome reveals the contribution of gut-kidney axis on kidney disease. J. Transl. Med. 2019, 17, 5. [CrossRef] [PubMed]

(C) 2019 by the authors. Licensee MDPI, Basel, Switzerland. This article is an open access article distributed under the terms and conditions of the Creative Commons Attribution (CC BY) license (http://creativecommons.org/licenses/by/4.0/). 